\title{
High-Sensitive Sensor of Dopamine Based on Photoluminescence Quenching of Hierarchical CdS Spherical Aggregates
}

\author{
Binjie Sun $^{1}$ and Changzheng Wang ${ }^{2}$ \\ ${ }^{1}$ College of Chemistry, Beijing Normal University, Beijing 100875, China \\ ${ }^{2}$ Key Laboratory of Urban Stormwater System and Water Environment, Beijing University of Civil Engineering and Architecture, \\ Beijing 100044, China
}

Correspondence should be addressed to Binjie Sun, sunbinjie@mail.bnu.edu.cn

Received 21 May 2012; Accepted 24 May 2012

Academic Editor: Xiao-Miao Feng

Copyright (c) 2012 B. Sun and C. Wang. This is an open access article distributed under the Creative Commons Attribution License, which permits unrestricted use, distribution, and reproduction in any medium, provided the original work is properly cited.

\begin{abstract}
Hierarchical CdS spherical aggregates have been fabricated by an assembling strategy starting from nanoparticles, which opens a general way to obtain hierarchical spherical aggregates of different types of materials. The hierarchical CdS spherical aggregates are of high porosity and high surface area, which give rise to unique photoluminescence properties. The desirable properties we report here will spur further developments of novel dopamine photoluminescence sensors based on the high surface area hierarchical CdS spherical aggregates fabricated with our unique assembling strategy. The novel dopamine photoluminescence sensor has a low detection limit of $1.0 \times 10^{-8} \mathrm{M}$, which is much lower than those reported previously.
\end{abstract}

\section{Introduction}

Dopamine (DA), as one of the most important neurotransmitters, was discovered in the 1950s, which has ever since captured the interest of neuroscientists and chemists. High DA levels are known to be cardiotoxic, leading to rapid heart rate, high blood pressure, and possible death of the heart muscles. On the contrary, a loss of DAcontaining neurons may result in some serious diseases such as Parkinson's disease and schizophrenia [1-7]. Hence, determining the concentration of this neurochemical is of biomedical significance.

The detection of DA is most often accomplished by the measurement of redox potential or intrinsic fluorescence. Fluorescence quenching refers to any process which decreases the fluorescence intensity of a sample, which has been widely studied both as a fundamental phenomenon and as a source of information about biochemical systems [8-10]. With the development of nanotechnology, many methods have been developed to detect DA by using nanomaterials such as carbon nanotubes and gold nanoparticles (NPs) [11-15]. Semiconductor nanoparticles have attracted much attentions since they have unique optical and several other properties such as size-dependent, tunable adsorption and emission properties [16]. Fluorescence detection methods have led to major improvement in bioanalytical applications because of their extraordinary sensitivity and selectivity. The traditional fluorescence probes are organic dyes, and semiconductor nanocrystals with unique structure could probably be used to substitute the organic dyes based on their unique optical properties.

As a unique structure, hierarchical assembly of nanoscale building blocks is a crucial step toward realization of functional nanosystems and represents a significant challenge in the field of nanoscience [17]. The past few years have witnessed increasing attention focused on such novel structures, because of their unique optical, electrical, and surface properties that underlie their potential applications in photoelectric devices, drug delivery, sensors, filters, coatings, and chemical catalysis. Methods that support hierarchical assembly over multiple length scales are particularly promising for designing functional nanostructures and assembling nanodevices. Many efforts have been developed to shape nanoscale materials to hierarchical nanoarchitectures, such as hierarchically ordered oxides (silica, niobia, and titania), zinc oxide hierarchically ordered nanowire arrays, 


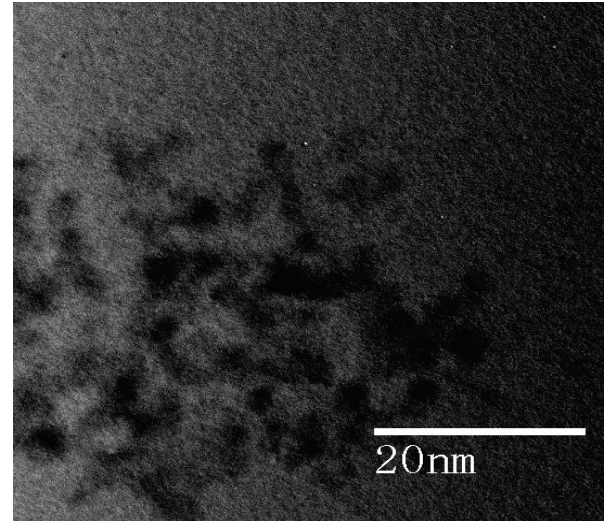

(a)

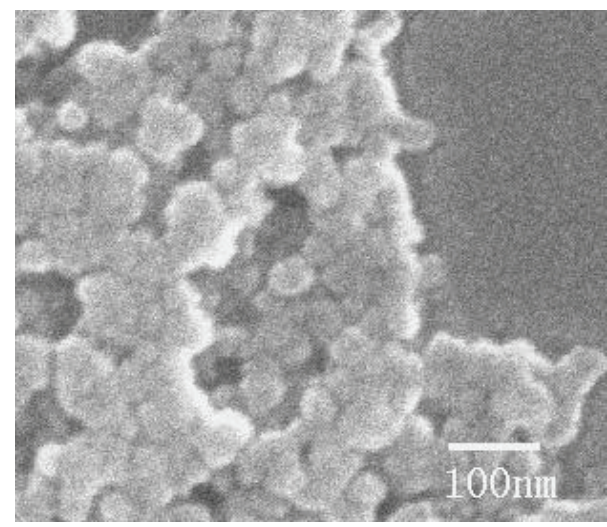

(c)

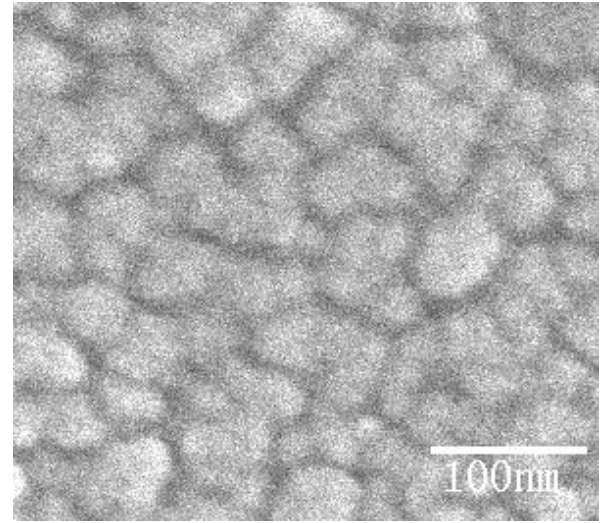

(b)

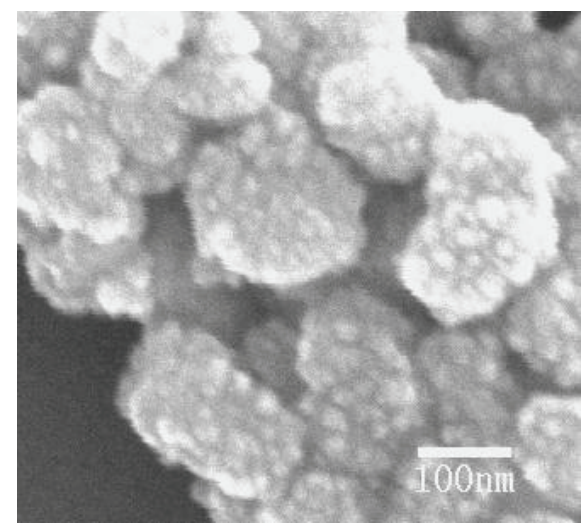

(d)

FIGURE 1: TEM image (a) of CdS colloids prepared in tetrachlorethylene and octylamine mixed solution and SEM images (b, c, d) of the hierarchical CdS spherical aggregates.

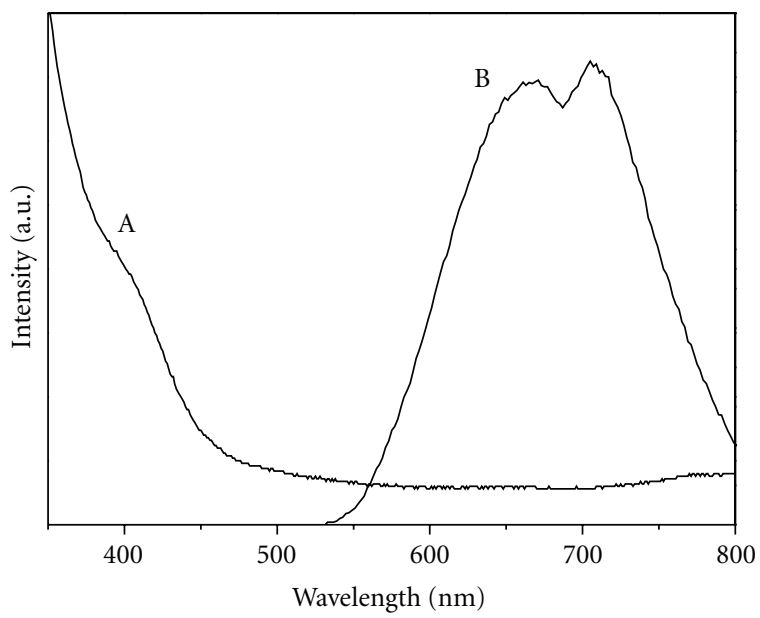

FIgURE 2: UV-vis absorption and photoluminescence spectra of hierarchical CdS spherical aggregates.

hierarchical zinc sulfide nanostructures, gold nanoparticle, nanotubes, and rhodium spherical aggregates [18-21].

Hierarchical spherical aggregates, who are composed of NPs in a multi-length-scale assembly, possess high surface area and therefore are particularly important in catalysis, sensors, and nanodevices. It is well known that CdS is an outstanding photoelectric semiconductor material [22, 23]. It is envisaged that NP-assembled hierarchical CdS spherical aggregates, with intimate inter-NP contacts and a direct connection to the electrode and large exposed surface areas, should provide an ideal nanoarchitecture for efficient generation of photoluminescence (PL). Although numerous shapes of CdS nanocrystals have been prepared, to our knowledge, there has been few reports on such hierarchical spherical aggregates from CdS NPs to be used as a sensor for DA. Herein, we report a simple approach for the detection of DA by high surface area hierarchical CdS spherical aggregates.

\section{Material and Methods}

All chemicals were of analytical grade and were used without further purification.

2.1. Preparation of Hierarchical CdS Spherical Aggregates. CdS NPs and their spherical aggregates were prepared in organic solvents, and the procedure is described here. CdS quantum dots were synthesized according to the literature 


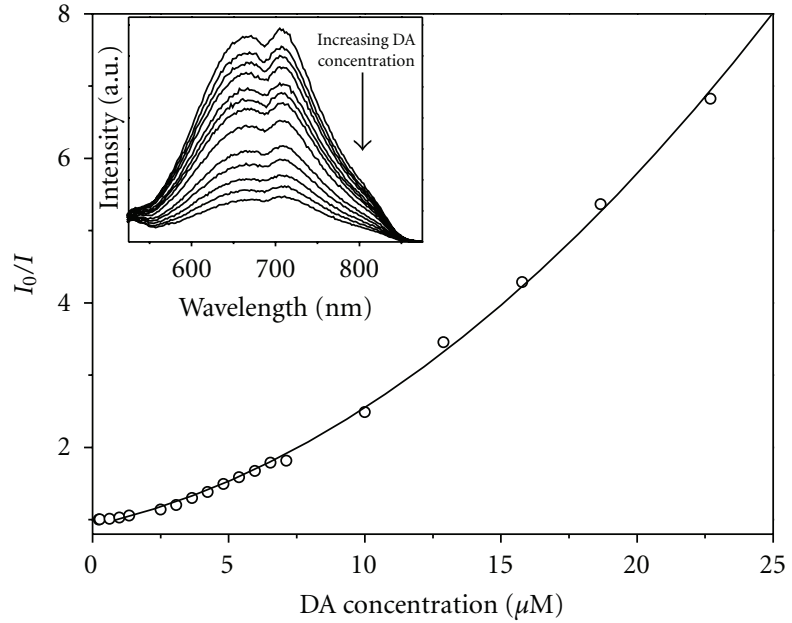

(a)

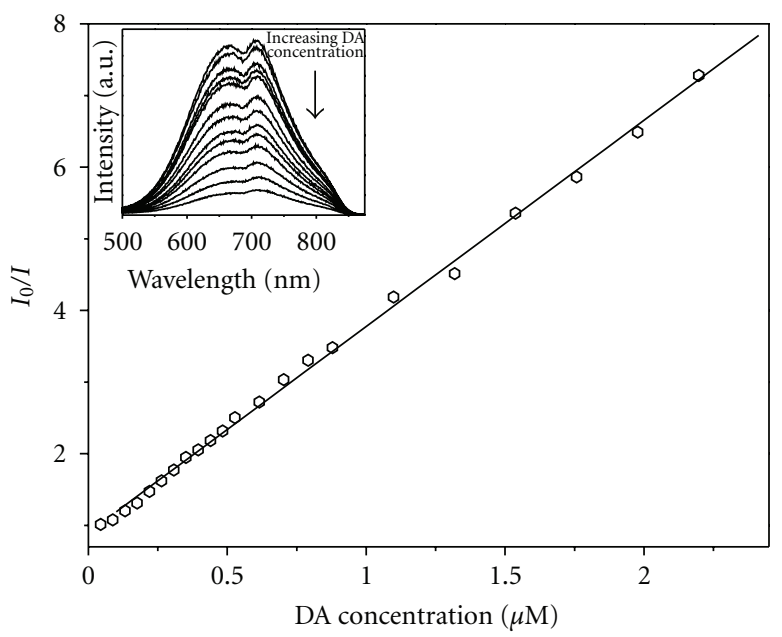

(c)

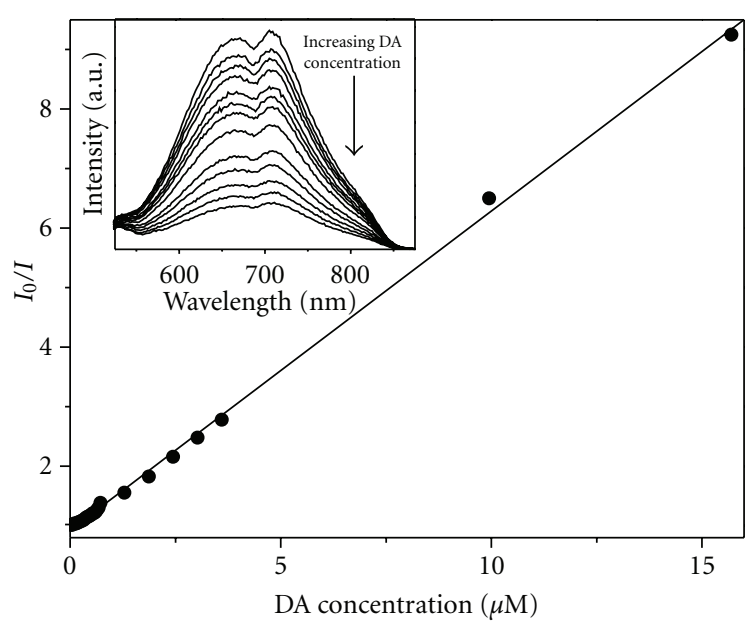

(b)

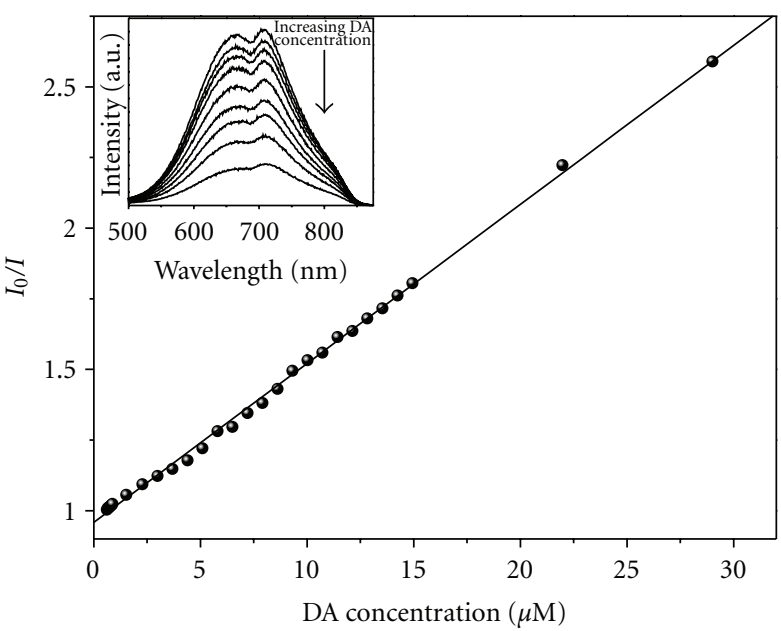

(d)

FIGURe 3: Dependence of the PL intensity of hierarchical CdS spherical aggregates on the concentration of DA, with different volume ratio of tetrachlorethylene to ethanol: (a) $4: 1$, (b) $1: 4$, (c) $1: 50$, and (d) CdS colloidal solution without ethanol.

but with improvement [24]. In a typical procedure, $0.25 \mathrm{~g}$ sublimed sulfur was dissolved in $200 \mathrm{~mL}$ dimethyl sulphoxide (DMSO) at $100^{\circ} \mathrm{C}$ for about 1 hour and then increased to $150^{\circ} \mathrm{C}$. At this temperature, a preheated solution of $2.5 \mathrm{~g}$ cadmium acetate in $200 \mathrm{~mL}$ DMSO was added. The solution became lemon yellow after several minutes. The reaction solution was kept in $150^{\circ} \mathrm{C}$ for about one hour and then cooled to ambient temperature. Adding $100 \mathrm{~mL}$ acetone to the solution, the precipitant was collected by centrifuging and then washed with acetone for two times. After dried under vacuum at room temperature, the CdS quantum dots were obtained. Analytical grade octylamine, which was used to increase the solubility of the CdS quantum dots, was dissolved in tetrachlorethylene (the volume ratio of octylamine to tetrachlorethylene was $0.1 \%$ ). CdS colloid in tetrachlorethylene was obtained through $0.1 \mathrm{mg} \mathrm{CdS}$ quantum dots adding $99.9 \mathrm{~mL}$ tetrachlorethylene and $0.1 \mathrm{~mL}$ octylamine. The CdS colloid was stable for more than 6 months at ambient temperature. Hierarchical CdS spherical aggregates were assembled by adding ethanol in the CdS colloid. The size distributions of these spherical aggregates vary with the volume ratio of tetrachlorethylene and ethanol.

2.2. The Photoluminescence Quenching of Hierarchical CdS Spherical Aggregates by Dopamine. DA was dissolved in ethanol. Hierarchical CdS spherical aggregates were dispersed in ethanol $(3 \mathrm{~mL})$, and the photoluminescence of CdS was recorded before DA was added. Then DA solution was dropped into CdS by injector, and after solution was mixed round adequately, the photoluminescence intensity was recorded step by step.

2.3. Characterization. The morphology and size of the hierarchical CdS spherical aggregates were analyzed with a Hitachi S4800 scanning electron microscope (SEM) and a Philips CM20 transmission electronic microscope (TEM). The UV-vis spectra were measured at room temperature with 


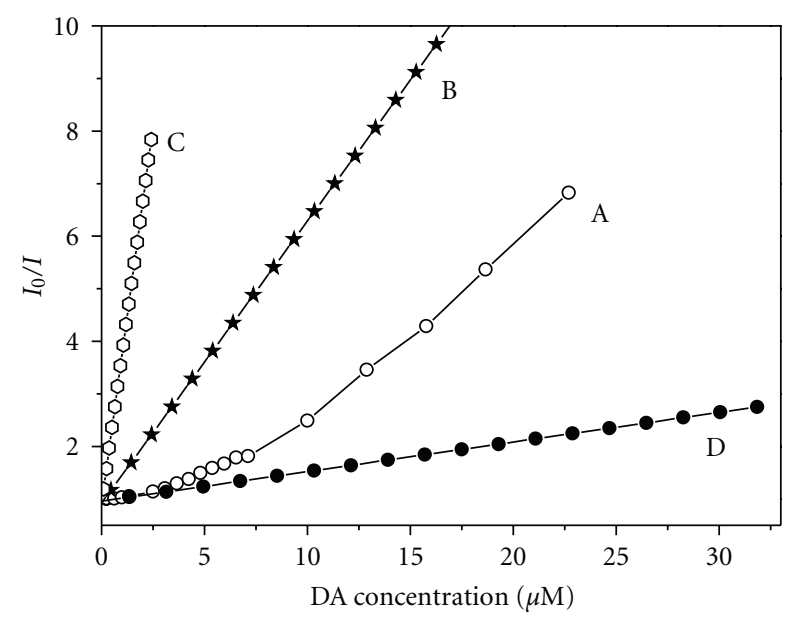

Figure 4: A linear relation between the PL intensity and DA concentration, with different volume ratio of tetrachlorethylene to ethanol: (a) 4:1, (b) $1: 4$, (c) $1: 50$, and (d) CdS colloidal solution without ethanol.

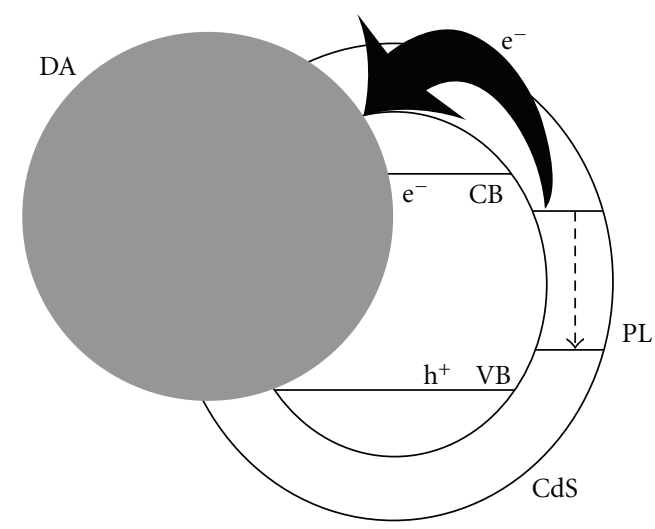

FIGURE 5: Schematic illustration for the fluorescence quenching principle of CdS in the presence of DA.

a lambda 900 UV-vis spectrophotometer (GBC, Cintra10). Photoluminescence spectra were measured at room temperature with an LS50 steady-state fluorescence spectrometer (Shimadzu, RF-5301PC).

\section{Results and Discussions}

TEM image (Figure 1(a)) shows that the diameter of the as-prepared CdS quantum dots is about $2-5 \mathrm{~nm}$, which dissolves neither in tetrachlorethylene nor in ethanol. When ethanol was added into the CdS colloid, the hierarchical CdS spherical aggregates could be obtained. SEM images testify the formation of hierarchical CdS spherical aggregates. Figures 1(b) to 1(d) show that as-prepared hierarchical CdS spherical aggregates are composed of small CdS NPs. The uniform spherical aggregates with diameter about $100 \mathrm{~nm}$ were built from CdS nanoparticles with the size of smaller than $5 \mathrm{~nm}$. The diameter of the spherical aggregates was controlled by the volume ratio of ethanol to tetrachlorethylene. With the increase of the volume ratio of ethanol to tetrachlorethylene, the diameter of the spherical aggregates increases.

From Figures 1(b) to 1(d) depicted the SEM images of hierarchical CdS spherical aggregates growing at different volume ratios of tetrachlorethylene to ethanol. Figure 1(b) showed the morphology of the sample, which is obtained when the ratio of tetrachlorethylene to ethanol is $4: 1(\mathrm{~V} / \mathrm{V})$. Its diameter ranges from 20 to $50 \mathrm{~nm}$. When this ratio was changed to be $1: 4(\mathrm{~V} / \mathrm{V})$, the diameter of spherical aggregates was larger as shown in Figure 1(c). As shown in Figure 1(d), when the ratio of tetrachlorethylene to ethanol was increased to $1: 50(\mathrm{~V} / \mathrm{V})$, the diameter of spherical aggregates ranges from 80 to $100 \mathrm{~nm}$, which were clearly comprised of bright spots of individual CdS quantum dots with the diameter of $20-30 \mathrm{~nm}$. But continuing to increase the volume ratio of tetrachlorethylene to ethanol, the CdS nanoparticles will be deposited. It distinctly indicated that the hierarchical CdS spherical aggregates were able to be produced by the self-assembly of CdS quantum dots, and the size of the self-assembled CdS spherical aggregates can be controlled by the volume ratio of tetrachlorethylene to ethanol.

Optical properties of the hierarchical CdS spherical aggregates have been studied by UV-vis absorption and photoluminescence (Figure 2). The UV-vis absorption spectra substantiate the formation of hierarchical CdS spherical aggregates (Figure 2(a)), which is consistent with the UVvis spectra of CdS nanoparticles, with the absorption peak $420 \mathrm{~nm}$. For hierarchical CdS spherical aggregates, the PL emissions are at around $700 \mathrm{~nm}$, which is attributed to the trapped emission (Figure 2(b)).

The effect of DA concentration on the PL emissions intensity of hierarchical CdS spherical aggregates is shown in Figure 3. The linear relation between the PL intensity and DA concentration can be obtained, respectively, when the ratio of tetrachlorethylene to ethanol is $4: 1,1: 4$, and $1: 50$.

As proved previously, addition of DA to the aqueous solution of CdS led to a significant quenching of the PL emission of CdS. Moreover, DA gave a steady and maximal quenching effect on the fluorescence of CdS. PL emission is much sensitive to their surface structures and is dependent on the surface properties and the presence of surface states. And, with much more ethanol concentration, the surface of hierarchical CdS spherical aggregates will be higher, and the quenching effect will become more prominent (Figure 4). When the ratio of tetrachlorethylene to ethanol is $1: 50$, the linear relation between the PL intensity and DA concentration was obtained. The detection limit of DA was detected to be $1.0 \times 10^{-8} \mathrm{M}$, lower than those of biosensors reported $[1,2]$, which demonstrate that hierarchical CdS spherical aggregates can be developed to be used as DA sensor.

The fluorescence quenching principle of CdS in the presence of DA is illustrated in Figure 5. The fact that DA substantially quenched the fluorescence of CdS indicates a strong interaction between the excited-state CdS and the attached DA molecules. A plausible explanation for the PL peak quenchments presented here is the unique surface electronic structure of the hierarchical CdS spherical aggregates. 
When the active sites of hierarchical CdS spherical aggregates increased, a junction is built up, and DA can act as an electron acceptor. Quenching of the PL emissions of CdS by DA is expected via the new nonradiative pathways created by the proximity of DA, possibly resulting from electron transfer from CdS to DA [25].

\section{Conclusions}

In summary, high surface area hierarchical CdS spherical aggregates have been fabricated by an assembling strategy starting from nanoparticles, which opens a general way to obtain hierarchical spherical aggregates of different types of materials. The hierarchical CdS spherical aggregates are of high porosity, high surface area, and high order, which give rise to unique PL properties. All of the desirable properties we report here will spur further developments of novel DA sensors based on the hierarchical CdS spherical aggregates fabricated with our simple assembling strategy.

\section{Acknowledgments}

The authors gratefully acknowledge the support from the National Nature Science Foundation of China (20975016, 21175014, 91027034, and 21103008), National Grant of Basic Research Program of China (no. 2011CB915504), the Fundamental Research Funds for the Central Universities (2009SC1), and Open Research Fund Program of Key Laboratory of Urban Stormwater System and Water Environment (Beijing University of Civil Engineering and Architecture), Ministry of Education.

\section{References}

[1] J. L. Chen, X. P. Yan, K. Meng, and S. F. Wang, "Graphene oxide based photoinduced charge transfer label-free near-infrared fluorescent biosensor for dopamine," Analytical Chemistry, vol. 83, no. 22, pp. 8787-8793, 2011.

[2] P. C. Pandey, D. S. Chauhan, and V. Singh, "Effect of processable polyindole and nanostructured domain on the selective sensing of dopamine," Materials Science and Engineering $C$, vol. 32, no. 1, pp. 1-11, 2012.

[3] C. Yu, J. Yan, and Y. Tu, "Electrochemiluminescent sensing of dopamine using CdTe quantum dots capped with thioglycolic acid and supported with carbon nanotubes," Microchimica Acta, vol. 175, no. 3-4, pp. 347-354, 2011.

[4] D. R. Shankaran, K. Limura, and T. Kato, "Simultaneous determination of ascorbic acid and dopamine at a sol-gel composite electrode," Sensors and Actuators B, vol. 94, no. 1, pp. 73-80, 2003.

[5] K. Kurzatkowska, E. Dolusic, W. Dehaen, K. Sieroń-Stołtny, A. Sieron, and H. Radecka, "Gold electrode incorporating corrole as an ion-channel mimetic sensor for determination of dopamine," Analytical Chemistry, vol. 81, no. 17, pp. 73977405, 2009.

[6] L. Lin, P. Qiu, L. Yang, X. Cao, and L. Jin, "Determination of dopamine in rat striatum by microdialysis and highperformance liquid chromatography with electrochemical detection on a functionalized multi-wall carbon nanotube electrode," Analytical and Bioanalytical Chemistry, vol. 384, no. 6, pp. 1308-1313, 2006.
[7] L. Zhang and X. Lin, "Electrochemical behavior of a covalently modified glassy carbon electrode with aspartic acid and its use for voltammetric differentiation of dopamine and ascorbic acid," Analytical and Bioanalytical Chemistry, vol. 382, no. 7, pp. 1669-1677, 2005.

[8] P. Bolel and M. Halder, "Fluorescence quenching of carmoisine by viologens in neat methanol: observation of inversion in quenching," Chemical Physics Letters, vol. 507, no. 4-6, pp. 234-239, 2011.

[9] B. Sui, L. Shen, and W. Jin, "Ultrasensitive determination of 1,4-dihydroxybenzene based on fluorescence resonance energy quenching of luminescent quantum dots modified on surface of silica nanoparticles," Talanta, vol. 85, no. 3, pp. 1609-1613, 2011.

[10] X. G. Gu, G. X. Zhang, and D. Q. Zhang, "A new ratiometric fluorescence detection of heparin based on the combination of the aggregation-induced fluorescence quenching and enhancement phenomena," Analyst, vol. 137, no. 2, pp. 365$369,2012$.

[11] H. Jeong and S. Jeon, "Determination of dopamine in the presence of ascorbic acid by nafion and single-walled carbon nanotube film modified on carbon fiber microelectrode," Sensors, vol. 8, no. 11, pp. 6924-6935, 2008.

[12] K. Wu, J. Fei, and S. Hu, "Simultaneous determination of dopamine and serotonin on a glassy carbon electrode coated with a film of carbon nanotubes," Analytical Biochemistry, vol. 318, no. 1, pp. 100-106, 2003.

[13] Z. H. Wang, Q. L. Liang, Y. M. Wang, and G. A. Luo, "Carbon nanotube-intercalated graphite electrodes for simultaneous determination of dopamine and serotonin in the presence of ascorbic acid," Journal of Electroanalytical Chemistry, vol. 540, pp. 129-134, 2003.

[14] C. R. Raj, T. Okajima, and T. Ohsaka, "Gold nanoparticle arrays for the voltammetric sensing of dopamine," Journal of Electroanalytical Chemistry, vol. 543, no. 2, pp. 127-133, 2003.

[15] H. P. Wu, T. L. Cheng, and W. L. Tseng, "Phosphate-modified $\mathrm{TiO}_{2}$ nanoparticles for selective detection of dopamine, levodopa, adrenaline, and catechol based on fluorescence quenching," Langmuir, vol. 23, no. 14, pp. 7880-7885, 2007.

[16] D. M. Willard, L. L. Carillo, J. Jung, and A. Van Orden, "CdSe$\mathrm{ZnS}$ quantum dots as resonance energy transfer donors in a model protein-protein binding assay," Nano Letters, vol. 1, no. 9, pp. 469-474, 2001.

[17] Y. Cheng, Y. Wang, C. Jia, and F. Bao, "MnS hierarchical hollow spheres with novel shell structure," Journal of Physical Chemistry B, vol. 110, no. 48, pp. 24399-24402, 2006.

[18] P. Yang, T. Deng, D. Zhao et al., "Hierarchically ordered oxides," Science, vol. 282, no. 5397, pp. 2244-2246, 1998.

[19] M. Lahav, T. Sehayek, A. Vaskevich, and I. Rubinstein, "Nanoparticle nanotubes," Angewandte Chemie, vol. 42, no. 45, pp. 5576-5579, 2003.

[20] T. D. Ewers, A. K. Sra, B. C. Norris et al., "Spontaneous hierarchical assembly of rhodium nanoparticles into spherical aggregates and superlattices," Chemistry of Materials, vol. 17, no. 3, pp. 514-520, 2005.

[21] A. D. Dinsmore, M. F. Hsu, M. G. Nikolaides, M. Marquez, A. R. Bausch, and D. A. Weitz, "Colloidosomes: selectively permeable capsules composed of colloidal particles," Science, vol. 298, no. 5595, pp. 1006-1009, 2002.

[22] D. Routkevitch, T. Bigioni, M. Moskovits, and J. M. Xu, "Electrochemical fabrication of CdS nanowire arrays in porous anodic aluminum oxide templates," Journal of Physical Chemistry, vol. 100, no. 33, pp. 14037-14047, 1996. 
[23] Y. Liang, C. Zhen, D. Zou, and D. Xu, "Preparation of freestanding nanowire arrays on conductive substrates," Journal of the American Chemical Society, vol. 126, no. 50, pp. 1633816339, 2004.

[24] R. Elbaum, S. Vega, and G. Hodes, "Preparation and surface structure of nanocrystalline cadmium sulfide (Sulfoselenide) precipitated from dimethyl sulfoxide solutions," Chemistry of Materials, vol. 13, no. 7, pp. 2272-2280, 2001.

[25] T. Mokari, E. Rothenberg, I. Popov, R. Costi, and U. Banin, "Selective growth of metal tips onto semiconductor quantum rods and tetrapods," Science, vol. 304, no. 5678, pp. 1787-1790, 2004. 

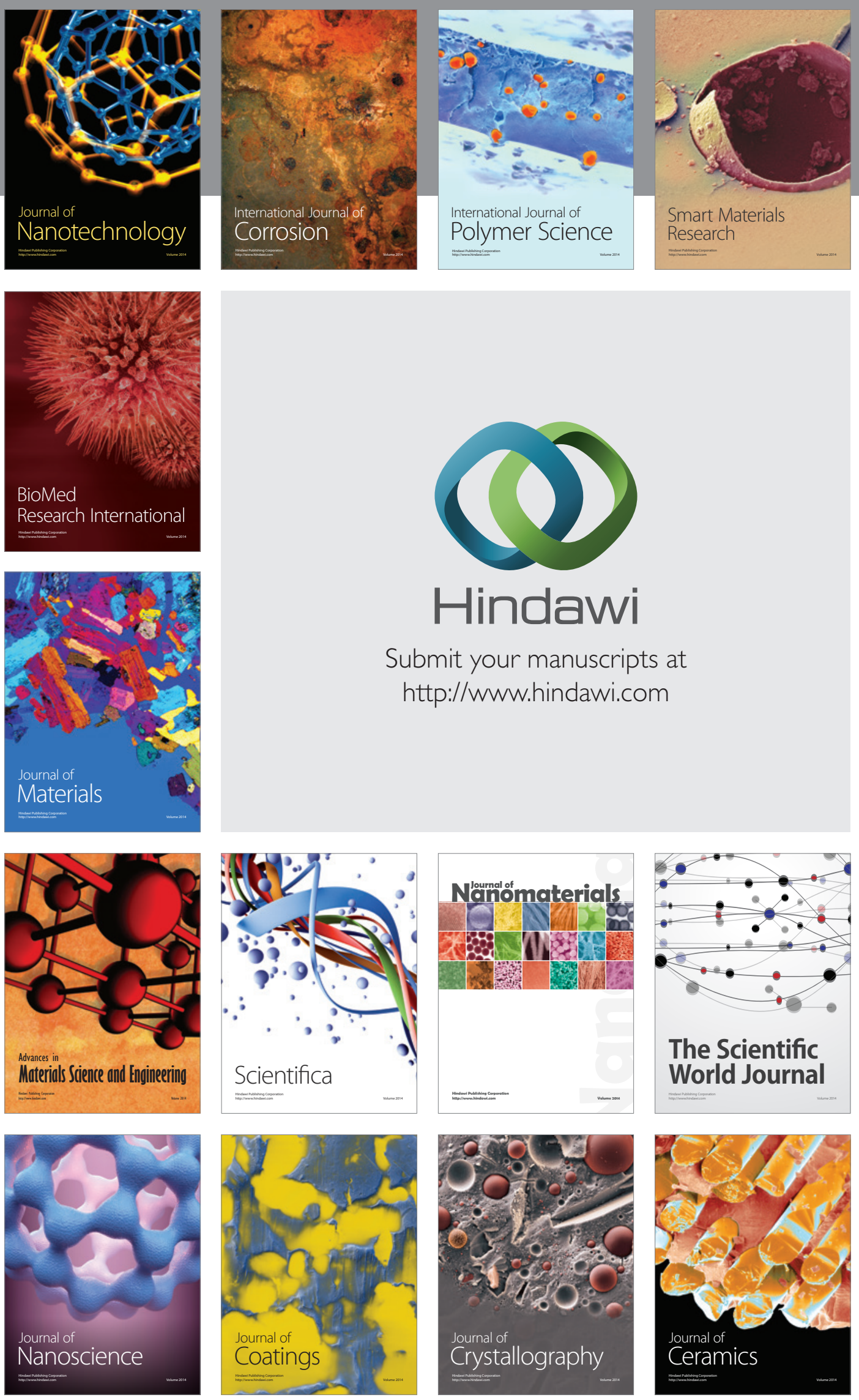

The Scientific World Journal

Submit your manuscripts at

http://www.hindawi.com

\section{World Journal}

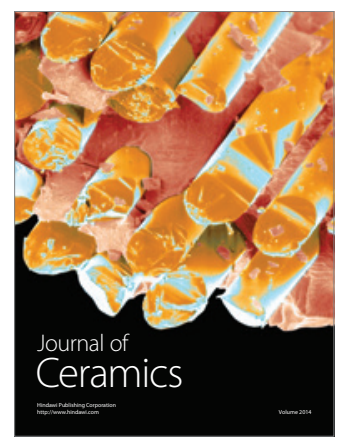

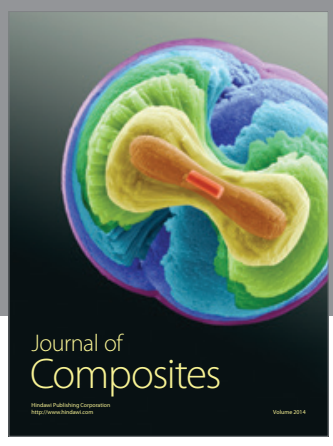
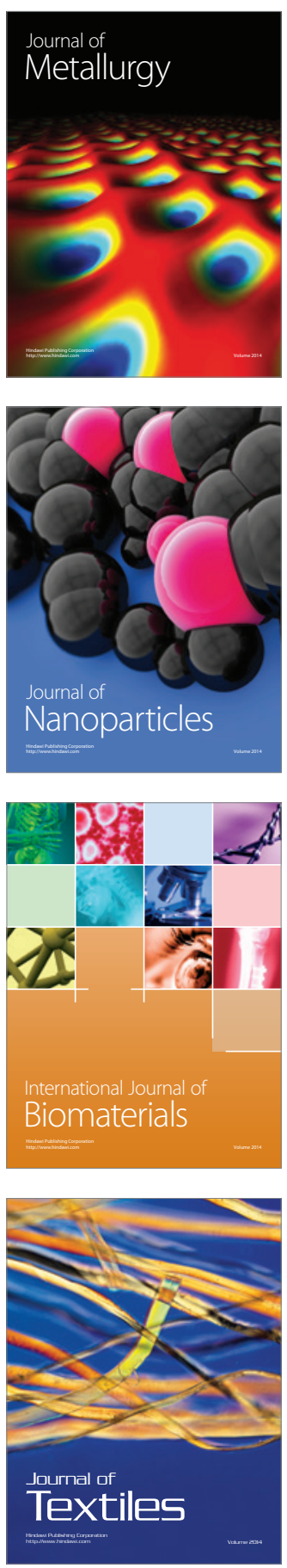